\title{
Igualdad de acciones afirmativas a mujeres que ingresan a la Función Judicial del
} Ecuador

\section{Equality of affirmative actions to women who enter the Judicial Function of Ecuador}

\author{
Diego Andrés Arpi-Silva \\ diego.arpi@psg.ucacue.edu.ec \\ Universidad Católica de Cuenca, Cuenca \\ Ecuador \\ https://orcid.org/0000-0002-7868-4153 \\ Cecilia Ivonne Narváez-Zurita \\ inarvaez@ucacue.edu.ec \\ Universidad Católica de Cuenca, Cuenca \\ Ecuador \\ https://orcid.org/0000-0002-7437-9880 \\ José Luis Vázquez-Calle \\ jlvazquezc@ucacue.edu.ec \\ Universidad Católica de Cuenca, Cuenca \\ Ecuador \\ https://orcid.org/0000-0003-4980-6403 \\ Juan Carlos Erazo-Álvarez \\ jcerazo@ucacue.edu.ec \\ Universidad Católica de Cuenca, Cuenca \\ Ecuador \\ https://orcid.org/0000-0001-6480-2270
}

Recibido: 13 de noviembre de 2019

Aprobado: 16 de diciembre de 2019 


\section{RESUMEN}

Este análisis, hace referencia a la acción afirmativa conforme lo establece el artículo 11 numeral 2 inciso tercero de la Constitución de la República del Ecuador (2008) en su parte medular: "El Estado adoptará medidas de acción afirmativa que promuevan la igualdad real en favor de los titulares de derechos que se encuentren en situación de desigualdad" (p.22). Creando una crítica notable en los postulantes que desean ingresar al sector público, que a su vez quebranta el principio de igualdad al beneficiar injustamente a las mujeres basándose en cuestiones que son ajenas a sus méritos y habilidades, siendo así que la medida de acción afirmativa no muestra ser injusta, sino que es poco factible que logre su propósito, por consiguiente, el objetivo de la investigación consiste en establecer si existe vulneración al principio de igualdad descrito en líneas anteriores.

Descriptores: Principio de igualdad; Desigualdad de género; Acción afirmativa; Desigualdad; Servicio público y mérito.

\section{ABSTRACT}

This analysis refers to affirmative action as established in article 11 number 2, third paragraph of the Constitution of the Republic of Ecuador (2008) in its core: "The State shall adopt affirmative action measures that promote real equality in favor of rights holders who find themselves in a situation of inequality "(p.22). Creating a notable criticism in the applicants who wish to enter the public sector, which in turn violates the principle of equality by unfairly benefiting women based on issues that are beyond their merits and abilities, so the affirmative action measure does not It shows to be unfair, but it is unlikely that it achieves its purpose, therefore, the objective of the investigation is to establish whether there is a violation of the principle of equality described in previous lines.

Descriptors: Principle of equality; Gender inequality; Affirmative action; Inequality; Public service and merit.

\section{INTRODUCCIÓN}

El Derecho Constitucional regula problemáticas concernientes a la sociedad y su funcionamiento, además establece los límites de dicha sociedad, porque a través de 
esto limita el actuar de un gobierno y crea una barrera frente a aquellas acciones que se encuentren fuera de la esfera constitucional, con el propósito de proteger a los seres humanos la no vulneración de los derechos conocidos como esenciales. En este sentido, el Derecho Constitucional nace con la finalidad de regular el poder político de una nación. Por su parte, el Neo constitucionalismo se constituye en una nueva concepción del Estado Constitucional de Derechos y Justicia. Es un elemento transformador, que está presente desde hace varias décadas en distintos países, incluido Ecuador. Se trata de una teoría jurídica de leyes que busca demarcar los poderes estatales y proteger los derechos fundamentales de las personas (Ávila, Salgado y Valladares, 2009; Ávila, 2012; Ávila, 2017).

Para Saba (2016) la "igualdad ante la ley significa igualdad de trato en igualdad de circunstancias" (p. 39). Es decir, el principio de igualdad se convierte en un derecho que todos los seres humanos poseen. Además, tiene como finalidad permitir que, los individuos disfruten de todos los derechos otorgados. La igualdad ante la ley implica que el Estado asumirá un rol importante, esto con el fin de garantizar a cada individuo igual acceso a la justicia en similares condiciones. Es cierto que existen características físicas, capacidades intelectuales y situaciones sociales diferentes, sin embargo, esto no significa que la diferencia entre las personas tenga que ser ventajosa para unos en todas las circunstancias (Masabanda Analuiza, Aman Llerena, Montero Solano \& Analuiza, 2019).

La acción afirmativa, por su parte, es un mecanismo establecido en la constitución para generar un acto de retribución hacia aquellos grupos que, por las iniquidades del pasado, han sido víctimas de desigualdades sociales. Esta preferencia se otorga a todas las personas consideradas como minorías, con la finalidad de compensar una historia de discriminaciones que las ha dejado en desventaja y, con ello, retribuir estas inequidades no deseadas a través de un mecanismo automático, que contribuya a la reducción de las desigualdades (Sandel, 2011). 
En la normativa ecuatoriana la acción afirmativa se encuentra plasmada en el art. 11, numeral 2, de la carta fundamental (2008) donde se ordena la aplicación de medidas de acción afirmativa a las personas sujetas de derechos que se encuentren en un entorno de desigualdad (Sandel, 2011). La problemática surge porque la aplicación de la acción afirmativa origina un conflicto en este tipo de concursos, pues favorecería a las mujeres, quienes se beneficiarían con base en criterios ajenos a sus cualidades y destrezas. No se pretende plantear que la medida de acción afirmativa sea injusta, sino que podría constituirse en una práctica perjudicial para los postulantes no incluidos en el grupo de personas en situación de desigualdad. En tal sentido, pasa a ser una acción que vulnera la igualdad establecida en la norma normarum.

Ante esta situación descrita la investigación tiene como objetivo: establecer si la medida de acción afirmativa aplicada a favor de las mujeres durante los concursos de méritos y oposición para la admisión a un cargo en la Función Judicial del Ecuador, vulnera el principio de igualdad establecido en la Constitución de la República. A su vez, este objetivo se bifurca en dos específicos: i) Realizar una aproximación doctrinaria al principio de igualdad y a la acción afirmativa establecidos en la normativa ecuatoriana, en la jurisprudencia constitucional y en los tratados internacionales; ii) efectuar un repaso histórico a las desigualdades económicas y sociales que conllevaron a la aplicación de la acción afirmativa.

\section{DESARROLLO}

\section{Principio de igualdad y la acción afirmativa en la normativa ecuatoriana}

La palabra igualdad genera inquietud entre los seres humanos ya que al momento de poner en práctica sus participaciones en los distintos sectores de la sociedad los coloca en un mismo punto de partida, no obstante, no implica que va a haber igualdad en los resultados (Begné, 2012). El artículo 11, numeral 2 de la Constitución de la República del Ecuador (2008) establece que: "Todas las personas son iguales y gozarán de los mismos derechos, deberes y oportunidades" (p.21). La norma constitucional prohíbe 
trasgredir o derogar el reconocimiento, goce o ejercicio de los derechos por el cual todas las personas son parte de una misma especie. Por su parte, la Declaración Universal de los Derechos Humanos (1948), establece: "Todos son iguales ante la ley y tienen, sin distinción, derecho a igual protección de la ley. Todos tienen derecho a igual protección contra toda discriminación que infrinja esta declaración y contra toda provocación a tal discriminación" (art. 7).

Es necesario indicar que la igualdad como un principio constitucional, puede presentar una doble esfera: formal cuando la ley brinda el derecho de ser tratados por iguales, lo que genera igualdad de condiciones y oportunidades a las personas, y material, que tiene que ver con la real posición social del individuo ante la sociedad. De esta manera, se eliminan obstáculos que impiden la participación en los ámbitos políticos, económicos, sociales y culturales (Eguiguren, 1997). Los dos tipos de igualdad poseen un mismo núcleo común: hacen referencia a la prohibición de discriminación y el respeto a la diferencia. Así, la primera asegura de igualdad de trato a todas las personas mediante una regla jurídica; que impide privilegios, mientras que la segunda no tiene que ver con situación social del sujeto en donde se aplica la ley para frenar iniquidades.

En consecuencia, se puede establecer que la igualdad formal da sentido a la seguridad jurídica; ello significa la prohibición normativa o legal de discriminar a una persona por sus rasgos físicos, psicológicos o culturales. Con este impedimento se garantizan los derechos humanos y de ciudadanía, colocándolos en el mismo punto de partida. Por el contrario, la igualdad material se relacionaría a las consecuencias. Por otra parte, la igualdad de género significa que los derechos, las responsabilidades y las oportunidades no dependen del sexo con el que se nace, si no que estos van a depender de los factores sociales para mejorar la vida de sus ciudadanos en igualdad de condiciones (Ruano, 2015). El Pacto Internacional de Derechos Civiles y Políticos (1976), establece que los Estados se obligan a garantizar la igualdad entre hombres y mujeres en el goce de todos sus derechos. 
En la antigüedad, la mujer ha sido considerada como una persona sin privilegios que hasta en la actualidad aún continúa a pesar de que la norma protege a los hombres y mujeres como iguales. Por ello, se ha planteado la eliminación de privilegios masculinos, paso esencial para transferir los privilegios asignados a las mujeres (Ávila, Salgado y Valladares, 2009). Ante lo expuesto, la única manera de beneficiar a las mujeres sería por medio de la acción afirmativa, que también discrimina al hombre, por no pertenecer a este grupo vulnerable, por lo cual es necesario seguir compensando las desiguales sociales con el fin de brindar un trato justo en equidad de género (Sentencia C-371/00, 2000).

\section{Las desigualdades como antesala a la acción afirmativa}

Las desigualdades se constituyen en un proceso histórico basado en diferenciaciones económicas, culturales, intelectuales, sociales o sexuales, que dan lugar a la discriminación. Rawls (1995) en relación a la desigualdad señala que los principios de justicia escogidos por las personas son dos: el uno exige igualdad en derechos, y dos considera que las personas no aventajadas solo serán compensadas si existe igualdad de condiciones. Rawls (1995) sugiere que la sociedad deberá atender a quienes tienen menos dones naturales y a quienes han nacido en las posiciones sociales menos favorables, para buscar una igualdad merecida. Es aquí, cuando aparece el concepto de acción afirmativa, López (2016) señala que implica un conjunto de instituciones que aseguran la accesibilidad al trabajo y que proporcionan cierto margen de acción que permita igualar las condiciones, así como reducir las distancias existentes, para los menos aventajados.

Sobre la compensación, López (2016) resalta que, si busca la justicia al interior de la sociedad, entonces será indispensable equilibrar las desventajas de índole social entre otros, mismos que influyen en la consecución de ciertos objetivos de vida. En tal sentido, si se persigue la nivelación en el campo del juego y la reducción del impacto de ciertas diferenciaciones en el punto de partida, pues se deberá recurrir a las acciones 
afirmativas, una de las estrategias para asegurar un apoyo social. El género, por su parte, es considerado como el origen de la desigualdad. Para Vizcarra (2008), la discriminación hacia mujeres deriva por las circunstancias sociales, personales y laborales siendo una respuesta a tales fenómenos discriminatorios, se deberá evitar la exclusión de las mujeres en la toma de decisiones políticas.

En los últimos años el sexo femenino como víctima de discriminación participa en programas de género con el fin de concientizar a la sociedad; sin embargo, "aún continúan excluidas como actoras sociales y sujetos libres, autónomos y autoreconocibles [...] siguen apareciendo sin posibilidad de corregir la posición subordinada en la que se encuentran" (Vizcarra, 2008, p. 144).

Como antecedente, las medidas de acción afirmativa han sido identificadas en el concepto de categorías sospechosas, empleadas cuando se consideran que existe una probable discriminación hacia ciertos grupos o personas en situaciones de vulnerabilidad, sea por raza, sexo condición, entre otros (Saba, 2008; Sentencia N. ${ }^{\circ}$ 292-16-SEP-CC, 2016); en este sentido, el Estado puede apelar a la categoría sospechosa para aminorar el efecto nocivo de las prácticas sociales que han ubicado a personas o grupos en posiciones desfavorables (Sentencia №7-11-IA/19, 2019). Por su parte, la acción afirmativa es empleada para compensar la discriminación histórica de grupos débiles, y al ser una medida de reparación inmediata, cumplido su fin se extinguen (Murillo, 2010).

Existen tres objeciones a las acciones afirmativas: i) resultan ineficientes por cuanto no existe activismo por parte de los grupos fuertes y débiles (López, 2016); ii) son ilegales porque delimitan las oportunidades a las personas que no forman parte del grupo limitado (Hellman y Moreau, 2013); y iii) dañan la autoestima de quien no resulta beneficiado (Nagel, 1981 citato por López, 2016).

La Convención sobre la eliminación de todas las formas de discriminación contra la mujer (1981) determina en su artículo 4, que la temporalidad de las medidas intenta acelerar la igualdad de facto entre hombres y mujeres. Estas medidas especiales 
buscan crear condiciones que impulsen la observancia de las mujeres en los ámbitos cultural, social, económico y estructural. Una vez logrado dicho avance, su aplicación debe cesar de manera inmediata. Lo que significaría que, si estas dos categorías de género otorgan y quitan privilegios, generaría equiparación entre los mismos.

Esto señala que las acciones afirmativas, tienen el propósito de rectificar el pasado en donde el sexo masculino tenía el poder y privilegios sobre las mujeres, sin embargo, de lo anotado hoy en día las mujeres ya no podrían pertenecer a este grupo beneficiado por estas medidas, ya que por su misma lucha histórica han logrado equiparar sus oportunidades a las de los hombres, tal como el acceso paritario a la educación en todos sus niveles, el acceso a cualquier puesto de trabajo e inclusive el actual logro de paridad en los cargos de elección popular; de otro modo se desvirtuaría el carácter de temporal que tienen las acciones afirmativas, convirtiéndose en una ventaja sin final.

\section{El servicio público y el mérito}

El propósito del servicio público es corregir las necesidades sociales, lo que se encuentra regulado bajo principios constitucionales y legales a través del Estado. El servidor público tiene una función social, pues es el vínculo entre el poder del Estado y la sociedad (Bautista, 2008). El acceso al servicio público debe hacerse de acuerdo a los principios de igualdad, mérito y capacidad.

La Constitución de la República del Ecuador (2008) en el artículo 331, establece que el Estado garantizará a las mujeres igualdad en la accesibilidad laboral, en la capacitación y en las promociones profesionales, así como en una remuneración equitativa. A su vez, se implementarán acciones adecuadas para la eliminación de las desigualdades, así, se cierra la posibilidad a toda forma de discriminación, acoso o acto de violencia que afecte a las mujeres en sus trabajos. Por su parte, el Código Orgánico de la Función Judicial (COFJ, 2009) determina que en los concursos para ingresar a la 
Función Judicial como para las acciones de promoción, se tomará en cuenta el principio de igualdad.

En lo que respecta al mérito, este se otorgará a las personas por su esfuerzo, trabajo y dedicación; de ahí que los individuos merecedores de esta virtud podrán optar por un mejor lugar de trabajo. Por lo tanto, la preparación profesional en el sector público genera una importancia indispensable, pues mediante la aplicación de aptitudes, destrezas y conocimientos se demostrará la idoneidad para desempeñar un cargo público y para administrar de una manera eficiente (Guerrero, 1998).

\section{¿La aplicación de las acciones afirmativas, vulnera el principio de igualdad?}

La norma fundamental del Ecuador, ha establecido al principio de igualdad como un acto de retribución hacia aquellos grupos que por las iniquidades del pasado han sido víctimas de desigualdades sociales, siendo importante verificar si la acción afirmativa como tal, se está llevando a cabo en la función judicial para los concursos públicos de méritos y oposición en donde podría estarse incumpliendo por las siguientes razones: La acción afirmativa generó dos puntos adicionales a las mujeres por el hecho de ser mujer, en el cual vulnera o no el principio de igualdad (Asamblea Constituyente, 2008). En Ecuador actualmente se trata de romper con la cruz de la desigualdad y la discriminación, a través de las acciones afirmativas, sobre todo en el ámbito laboral, frente a lo cual se ha planteado una serie de estrategias que contribuyen a que todos los ciudadanos puedan tener las mismas oportunidades, entre esas a las mujeres que han recibido dos puntos.

De lo expuesto en líneas anteriores, se aprecia que unos ciudadanos gozan de mayores privilegios que otros, evidenciándose que las oportunidades de las personas para realizar sus potenciales individuales dependen de otros factores sociales sujetos a cambio; se puede indicar que se pretende llegar al principio de igualdad a través de las acciones afirmativas, en base a los mismos derechos y oportunidades, porque les dan ciertos beneficios a las mujeres por el simple hecho de serlo ante una situación real e 
histórica o encontrarse en desventaja respecto de la sociedad, generando una preferencia al momento de concederles una puntuación extra en los concursos de méritos y oposición (Figueroa, 2016). Partiendo de esa hipótesis se puede considerar que la aplicación de las acciones afirmativas en sí misma crea situaciones de desventajas en contra de las personas que no necesitan dichas acciones por no pertenecer a ningún grupo desventajado. De aquello la acción afirmativa no solo estaría vulnerando el principio de igualdad contemplado en la constitución, sino que también estaría atentando el derecho al trabajo del cual brinda una vida digna y decorosa en beneficio de los ciudadanos por igual.

En este marco de referencia, la jurisprudencia estadounidense cuando incorporó a las categorías sospechosas lo hizo considerando asuntos de raza, origen nacional y de extranjería siendo una serie de desventajas históricas, en tal sentido, si la acción afirmativa se aplica en Ecuador se estaría adoptando un concepto que fue creado para otro tipo de problemas, aquí cabe la pregunta ¿Se podría considerar al género dentro de una categoría sospechosa? Otra pregunta ¿Se podría considerar al género mujer como una situación en desventaja que merece una acción afirmativa que la respalde con puntos o beneficios?, creando así una duda en cuanto a la constitucionalidad de las acciones afirmativas previstas en el último inciso del artículo 11 numeral 2 de la constitución (Asamblea Constituyente, 2008), por cuanto se estarían otorgando ventajas, beneficios o privilegios a personas con fundamento en categorías sospechosas, cuya utilización genera una duda cuestionable y que debería especificarse que categorías debería ser sospechosas o no.

En el presente análisis, es necesario considerar lo que ha determinado la propia Constitución de la República del Ecuador, así como la Ley Orgánica de Servicio Público, la primera señala como obligación que: cualquier persona que quiera acceder a un cargo público debe hacerlo a través de un concurso de méritos y oposición, para ello debe contar con el perfil requerido para dicho puesto, a su vez con la capacidad solicitada (Asamblea Constituyente, 2008). Por su parte, la Ley Orgánica de Servicio 
Público (2010) adiciona que para ingresar al sector público debe seguir los concursos establecidos, donde tendrá más peso la idoneidad de los postulantes basados en criterios de justicia, transparencia y no discriminación, los cuales son herramientas cruciales para la generación de igualdad en la sociedad, en tanto que, el COFJ (2009) establece que para la admisión a esta institución se lo hará a través de concurso que dará atención primordial a los principios de igualdad y no discriminación.

En este contexto, también es necesario revisar lo que señala el Reglamento de Selección y Designación de Servidores de la Función Judicial (2011), al respecto estipula que las acciones afirmativas son aplicables en base a puntos y que las mismas son acumulables hasta 4 a favor de la mujer. Frente a este escenario, es ineludible preguntarse: ¿Qué pasa si una persona de sexo masculino en base a sus conocimientos, trayectoria y profesionalismo ha obtenido un puntaje de 92 sobre 100 frente a otra persona que es mujer quien ha obtenido 90 puntos? En respuesta a esta pregunta se podría inferir que la persona a quien se le asignó 2 puntos mediante la acción afirmativa no brinda un perfil tan excelente como el primero, significaría entonces, que esos dos puntos pueden crear la posibilidad de que las personas que acceden a un cargo público bajo este parámetro no sean las más calificada, sino más bien que por el hecho de ser mujer situación que no tiene que ver con la capacidad de formación ha obtenido el puesto.

Ante lo manifestado, hay que reflexionar que, al favorecer a ciertas personas en desmedro de otros, se podría afectar de manera directa la calidad del servicio público que la sociedad merece recibir. Por ello se requiere salir desde un mismo punto de partida en igualdad de condiciones para todos los participantes, con el fin de garantizar el derecho que tienen las personas a recibir un servicio estatal eficaz, eficiente y de calidad que satisfaga las necesidades de la sociedad; y esto solo se logra teniendo servidores públicos probos, preparados y capacitados para ocupar un puesto público de tanta relevancia como es la administración de justicia. 
Un aspecto a considerar y que va más allá del principio de igualdad, es que, al momento de otorgar una acción afirmativa a favor de las mujeres por el hecho de serlo, se estaría asumiendo que existe un menoscabo por haber tenido un pasado histórico de discriminación, como no se aclara el asunto contradice la manera de entender que el género femenino no podría obtener un cargo público por sus capacidades y méritos.

A modo de conclusión se ha determinado que la aplicación de la acción afirmativa en el caso puntual descrito vulnera el principio de igualdad constitucional, lo que a su vez significa el incumplimiento o la implicación de ciertos derechos constitucionales, en tal sentido, no se respeta el derecho a la igualdad ni se garantiza la participación de los ciudadanos en igualdad de condiciones. Por ello es indispensable que el Estado ecuatoriano promueva e incentive el interés de los ciudadanos por superarse cada día en el ámbito profesional, superación que les va a permitir mejores oportunidades en el ámbito laboral; contribuyendo así con el desarrollo del país al tener profesionales que en el ejercicio de sus funciones garanticen la prestación de servicios judiciales en un nivel óptimo, como se observa en la figura 1.

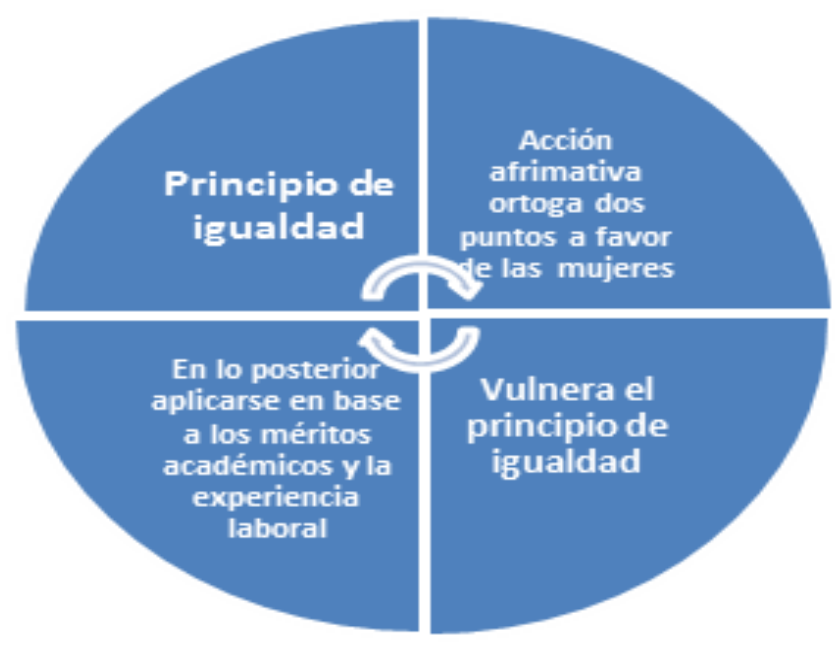

Figura 1. Incumplimiento del principio de igualdad en la aplicación de acciones afirmativas a mujeres que ingresan a la Función Judicial. 


\section{METODOLOGÍA}

La metodología utilizada en la presente investigación se sustentó en los métodos: analítico-sintético, lo que permitió analizar, normas jurídicas en base al tema planteado, descomponiendo sus elementos, para a partir de su reintegración, encontrar la relación entre las partes y el todo, así como se utilizó el método inductivo-deductivo, por cuanto el estudio se basó en cuestiones concretas, esto es el estudio de la aplicación de las acciones afirmativas a favor de las mujeres en los concursos públicos de la función judicial en base a los Reglamentos de Concursos de Méritos y Oposición, Impugnación Ciudadana y Control Social para el Ingreso a la Función Judicial, por lo que se obtuvo conclusiones generales; del mismo modo, se aplicó el método histórico-lógico, por cuanto se realizó un estudio al principio de igualdad y la acción afirmativa considerando su trayectoria y los acontecimientos que han propiciado su evolución en el decurso del tiempo (Lindao, Narváez y Erazo, 2019).

\section{APORTES}

El principio de igualdad busca suprimir diferencias de la especie humana, así como privilegios o exclusiones para favorecer a otros, ya sea por situaciones de raza, sexo o situación económica entre otras. Al remitirse a la Constitución de la República del Ecuador (2008) su texto consagra principios y obligaciones que han sido objeto de un gran estudio doctrinario, tanto en la normativa nacional, así como internacional, en donde se han implementado los avances históricos en reconocimiento a la igualdad de género en igualdad de condiciones; la misma que implica una condición que no reconoce diferencias entre seres humanos.

En lo referente a las acciones afirmativas contempladas en el mismo marco constitucional, éstas fueron creadas para brindar un trato preferencial a personas que han sufrido discriminación ante la sociedad, por ello es evidente que hay un reconocimiento hacia las mujeres por ser consideradas como un grupo de categoría inferior. Sin embargo, la aplicación de estos mecanismos en los concursos para 
acceder a un cargo público como es el caso de la Función Judicial en Ecuador, están vulnerando el derecho a la igualdad, conforme lo contempla la normativa ecuatoriana y Tratados y Convenios Internacionales en favor de los derechos humanos.

Por otra parte, la adopción de estas medidas de acción afirmativa en el ya citado reglamento que regula la puntuación adicional, vulnera el derecho de igualdad y partición al otorgar puntaciones extras a favor de las mujeres, siendo irrazonable que se otorguen dos puntos y que estos puedan ser acumulables hasta cuatro, por el hecho de ser mujer y considerarse en situación de desventaja y vulnerabilidad, y más aún es objetable el hecho que ese puntaje extra se les conceda en la fase de méritos, afectando de manera directa la calidad del servicio judicial que la sociedad merece recibir por no considerar la preparación profesional como las capacidades de los postulantes.

En definitiva, si la acción afirmativa vulnera el principio de igualdad, también considera a las mujeres como personas de menor capacidad intelectual, al no poder competir en los concursos para ocupar un cargo público en el servicio de justicia en igualdad de condiciones; lo que significa una ofensa a su capacidad y a sus propios méritos, por cuanto se puede pensar que las mujeres al ser beneficiadas por estas medidas no pueden obtener un cargo público por sí mismos.

Para ello es necesario, que la administración de justicia y de sus operadores, brinden los servicios de forma adecuada, respetando los principios de eficacia, eficiencia y calidad. Además, se considera relevante que los mencionados concursos se funden en los méritos y capacidades de los aspirantes a servidores públicos, mediante una selección y designación transparente tal como lo determina la Constitución del Ecuador. Bajo estas consideraciones, se plantea que las medidas de acción afirmativa a favor de las mujeres, en los concursos de mérito y oposición de la Función Judicial se apliquen en base a los méritos académicos y la experiencia laboral. 


\section{REFERENCIAS CONSULTADAS}

1. Asamblea General de las Naciones Unidas. (1948). Declaración Universal de Derechos Humanos. París.

2. Asamblea Nacional Constituyente. (2008). Constitución de la República del Ecuador. Montecristi, Ecuador.

3. Ávila Santamaría, R., Salgado, J., y Valladares, L. (2009). El género en el derecho. Quito: V\&M Gráficas.

4. Ávila, R. (2009). El constitucionalismo ecuatoriano. Breve caracterización de la Constitución de 2008 México: Instituto de Investigaciones Jurídicas. En Carbonell, M.; Carpizo, J.; Zovatto, D., Tendencias del Constitucionalismo en Ibero América (págs. 953-980). UNAM.

5. Ávila, L. (2017). Garantismo y estado constitucional en Estado de Ecuador para el siglo XXI. A propósito de Principia luris. Ciencia Jurídica, 5(10), 37-71.

6. Ávila, R. (2012). En defensa del neoconstitucionalismo transformador: los debates y los argumentos. (Universidad Andina Simón Bolívar) Recuperado de http://repositorio.uasb.edu.ec/bitstream/10644/2922/1/\%C3\%81 vila\%2C\%20RCON-004-En\%20defensa.pdf

7. Begné, P. (2012). Acción afirmativa: una vía para reducir la desigualdad (Vol. 1). Ciencias Jurídicas.

8. Código Orgánico de la Función Judicial (Registro Oficial №544 ed.). (2009). Quito.

9. Eguiguren, F. (1997). Principio a la igualdad y derecho la no discriminación. ius et veritas, 8(15), 63-72.

10. Figueroa, R. (2016). Acción Afirmativa en la Jurisprudencia del Tribunal Constitucional. Revista chilena de derecho, 401-433.

11. Guerrero, O. (1998). El funcionario, el diplomático y el juez. México: Plaza y Valdés Editores.

12. Hellman, D., y Moreau, S. (2013). Fundamentos filosóficos del derecho de discriminación. Oxford: OUP Oxford.

13. Lindao Bravo, D. S., Narváez Zurita, C. I., \& Erazo Álvarez, J. C. (2019). La contabilidad de gestión estratégica como herramienta multidisciplinar de planificación y control en la exportadora bananera Novamerc S.A. Koinonía, 165. 
14. Ley Orgánica de Servicio Público (Registro Oficial № 294 ed.). (2010). Quito.

15. Masabanda Analuiza, G., Aman Llerena, A., Montero Solano, J., \& Javier Analuiza, E. (2019). La evolución del derecho laboral de la mujer. Protección y fomento de la igualdad. IUSTITIA SOCIALIS, 4(7), 64-96. doi:http://dx.doi.org/10.35381/racji.v4i7.355

16. Murillo, P. (2010). Las medidas de acción afirmativa o medidas o especiales: para reparar

17. Reglamento de Concursos de Méritos y Oposición, Impugnación Ciudadana y Control Social para el Ingreso a la Función Judicial (Registro Oficial №293 ed.). (2014).

18. Organización de las Naciones Unidas. (2 de junio de 1981). Convención sobre la eliminación de todas las formas de discriminación contra la mujer. Convención sobre la eliminación de todas las formas de discriminación contra la mujer. San Salvador, República de El Salvador

19. Sentencia №7-11-IA/19, №7-11-IA/19 (Corte Constitucional dEcuador 28 de octubre de 2019).

20. Pacto Internacional de Derechos Civiles y Políticos. (1976). Pacto Internacional de Derechos Civiles y Políticos.

21. Rawls, J. (1995). Teoría de la justicia. México D.F.: Fondo de Cultura Económica.

22. Ruano, A. (16 de diciembre de 2015). La igualdad de género en la Función Pública del Estado ecuatoriano. Revista Ciencia UNEMI, 6(16), 107-119.

23. Saba, R. (2008). Igualdad, Clases y Clasificaciones: Buenos Aires: Roberto Gargarella, Teoría y Critica del derecho Constitucional, Tomo II, Abeledo Perrot.

24.Sandel, M. (2011). Justicia ¿Hacemos Lo que debemos? Barcelona, España: Random House Mondadori.

25. Saba, R. (2016). Más allá de la igualdad formal ante la ley. Buenos Aires: Siglo Veintiuno Editores Argentina S.A.

26. Vizcarra, I. (2008). Entre las desigualdades de género: un lugar para las mujeres pobres en la seguridad alimentaria y el combate al hambre. (México, DF), Argumentos, 21(57), 141-173.

27. Sentencia C-371/00, Expediente P.E.010 (Corte Constitucional de Colombia 29 de marzo de 2000). 
Iustitia Socialis. Revista Arbitrada de Ciencias Jurídicas.

Año V. Vol. V. N¹. Edición Especial. 2020

Hecho el depósito de Ley: FA2016000064

ISSN: 2542-3371

FUNDACIÓN KOINONIA (F.K). Santa Ana de Coro, Venezuela

Diego Andrés Arpi-Silva; Cecilia Ivonne Narváez-Zurita; José Luis Vázquez-Calle; Juan Carlos Erazo-Álvarez

\section{Sentencia N. ${ }^{\circ}$ 292-16-SEP-CC, Caso N. ${ }^{\circ}$ 0734-13-EP (Corte Constitucional del Ecuador 7 de septiembre de 2016).}

(C2020 por los autores. Este artículo es de acceso abierto y distribuido según los términos y condiciones de la licencia Creative Commons Atribución-NoComercial-Compartirlgual 4.0 Internacional (CC BY-NC-SA 4.0)

(https://creativecommons.org/licenses/by-nc-sa/4.0/). 